doi: http://dx.doi.org/10.7124/bc.0009A6

\section{TPR, chromatin organization, gene expression and cell development}

Jana Uhlířová ${ }^{1,4}$, Jindřiška Fišerová1,4, Karel Fišer ${ }^{2}$ and Pavel Hozák ${ }^{1,3,4}$

\footnotetext{
${ }^{1}$ Department of Biology of the Cell Nucleus, Institute of Molecular Genetics CAS, v.v.i., Vídeňská 1083, Prague, Czech Republic; ${ }^{2}$ CLIP-Childhood Leukaemia Investigation Prague, Department of Pediatric Hematology/Oncology; ${ }^{2}$ Medical Faculty of the Charles University and University Hospital Motol, V Úvalu 84, Prague, Czech Republic; ${ }^{3} \mathrm{Mi}-$ croscopy Centre - LM and EM, Institute of Molecular Genetics CAS, v.v.i., Vídeňská 1083, Prague, Czech Republic; ${ }^{4}$ Division BIOCEV, Institute of Molecular Genetics CAS, v.v.i., Průmyslová 595, Vestec, Prague, Czech Republic jana.uhlirova@img.cas.cz
}

The area around nuclear pores is enriched in open chromatin. Transcriptional factors and super enhancer sequences localize there during cell differentiation, activating sets of genes specific for a given tissue. Nucleoporin TPR is crucial for the enrichment of open chromatin around nuclear pores, however its role in gene expression and cell development has not been described yet. Here we show that depletion of TPR results in aberrant morphology of murine proliferating $\mathrm{C} 2 \mathrm{C} 12$ myoblasts $(\mathrm{MB})$ and differentiated $\mathrm{C} 2 \mathrm{C} 12$ myotubes (MT). Our CHIP-seq data revealed that TPR binds to Myosin heavy chain (Myh) gene and majority of olfactory receptor (Olfr) genes in $\mathrm{C} 2 \mathrm{C} 12$ $\mathrm{MB}$, and its binding pattern changes upon differentiation into MT. Both Myh and Olfr are expressed in muscle cell and regulate the muscle formation and morphology. We show that TPR affects expression of both Myh and Olfr genes, however in a different manner. Here we discuss possible pathways by which TPR regulates expression of bound genes.

This work was supported by the GACR (15-08835Y, 1603403S, 18-19714S), GAUK (930218) and by the institutional support (RVO: 68378050). I acknowledge the Microscopy Centre funded by MEYS CR (LM2015062, Czech-Biolmaging) for their support.

doi: http://dx.doi.org/10.7124/bc.0009A7

\section{Effects of selective degradation of the cohesin complex on higher order chromatin structures studied with live cell and super- resolved fluorescence microscopy}

Marion Cremer, Katharina Brandstetter, Andreas Maiser, Heinrich Leonhardt, Hartmann Harz,

Thomas Cremer Biocenter, Department Biology II, Ludwig Maximilians-University (LMU), Germany Current models postulate that cohesin is essential for the organization and shaping of the genome into chromatin loops/domains 1 . Hi-C data based on large cell populations showed that cohesin loss eliminates all loop domains 2. Here we aimed to investigate the effect of cohesin loss on the preservation/alteration of higher order chromatin structures on the single cell level using super-resolution microscopy and live cell studies. As reference structures we chose replication domains (RDs), first detected in S-phase nuclei as replication foci. RDs stably persist as basic units of higher order chromatin domains (CDs) throughout interphase and during subsequent cell cycles 4 . Methods \& 
Results All experiments were performed with HCT-116-RAD21-mAID-mClover cells, where degradation of cohesin is achieved by auxininduced proteolysis of its subunit RAD21 6. In live cell studies we demonstrated the appearance of mitotic cells up to $\sim 16 \mathrm{~h}$ after cohesin degradation with a strongly prolongated mitotic phase and final transition into one multilobulated nucleus. Typical RD patterns, achieved by replication-scratch labeling, persisted after cohesin degradation at least up to $46 \mathrm{~h}$ and were transmitted over mitosis irrespective of highly abnormal nuclear morphologies. No significant difference of RD patterns and size of individual RDs was noted at the resolution level of 3D-SIM between control cells and cells, which were replication-labeled before cohesin degradation. By contrast, when replication-labeling was performed after cohesin degradation, we noted a reduced number and coarsening of RD structures. Conclusions Our microscopic observations provided information on the effects of a cohesin degradation in individual cells, which were not recognized in $\mathrm{Hi}-\mathrm{C}$ studies of entire cultures. These findings exemplify the importance to combine $\mathrm{Hi}-\mathrm{C}$ with advanced 3D and 4D microscopy. Single cell $\mathrm{Hi}-\mathrm{C}$ studies of individual interphase and mitotic cells identified by microscopy could help to further study connections between the level of chromatin loops and higher order chromatin arrangements in interphase and mitosis following cohesin depletion.

References: 1 Nishiyama, T. Curr Opin Cell Biol 58, 8-14, doi:10.1016/j.ceb.2018.11.006 (2018). 2 Rao, S. S. P. et al. Cell 171, 305-320 e324, doi:10.1016/j.cell.2017.09.026 (2017). 3 Bintu, B. et al. Science 362, doi:10.1126/science. aau1783 (2018). 4 Cremer, T. et al. Curr Opin Cell Biol 18, 307-316, doi:10.1016/j.ceb.2006.04.007 (2006). 5 Pope, B. D. et al. Nature 515, 402-405, doi:10.1038/nature13986 (2014). 6 Natsume, T. Cell Rep 15, 210-218 (2016).

\section{WORKSHOP I: NUCLEAR STRUCTURE - CHROMATIN II}

doi: http://dx.doi.org/10.7124/bc.0009A8

\section{Determinants of nucleosome stability}

Laszlo Imre, Peter Nanasi, Rosevalentine Bosire, Erfaneh Firouzi Niaki and Gabor $\underline{\text { Szabo }}$

Dept. Biophysics and Cell Biology, University of Debrecen, Hungary szabog@med.unideb.hu

Nucleosome structure is, in general, repressive; hence, stability of nucleosomes is of regula- tory importance in eukaryotes. Recently, we have developed a quantitative, laser scanning cytometric approach (1) to characterize nucleosome stability in situ, in a cell cycle-, posttranslational modification-, and histone variantspecific manner. This approach has allowed us to assess how the above actors, as well as superhelical density, the presence of certain reader proteins and other molecular associations regulate this central player of transcriptional regulation. Through the spectacles of nucleosome stability, a simple link can be established between the superimposed levels of chromatin 